\title{
Myelin Oligodendrocyte Glycoprotein-Independent Rubella Infection of Keratinocytes and Resistance of First-Trimester Trophoblast Cells to Rubella Virus In Vitro
}

\author{
Quang Duy Trinh * (D), Ngan Thi Kim Pham, Kazuhide Takada, Shihoko Komine-Aizawa \\ and Satoshi Hayakawa * (iD \\ Division of Microbiology, Department of Pathology and Microbiology, Nihon University School of Medicine, \\ Tokyo 173-8610, Japan; pham.thikimngan@nihon-u.ac.jp (N.T.K.P.); rsa15836@nifty.com (K.T.); \\ aizawa.shihoko@nihon-u.ac.jp (S.K.-A.) \\ * Correspondence: trinh.duyquang@nihon-u.ac.jp (Q.D.T.); hayakawa.satoshi@nihon-u.ac.jp (S.H.); \\ Tel.: +81-3972-8111 (ext. 2261) (Q.D.T.)
}

Received: 8 December 2017; Accepted: 3 January 2018; Published: 4 January 2018

\begin{abstract}
Rubella virus (RuV), which belongs to the family Togaviridae and genus Rubivirus, causes systemic infection in children and young adults and congenital rubella syndrome in developing fetuses if the infection occurs during pregnancy. The mechanisms of fetal infection by RuV are not completely understood. Myelin oligodendrocyte glycoprotein (MOG) is reported to be a cellular receptor for $\mathrm{RuV}$; however, it is mainly expressed in the central nervous system. Therefore, it is thought that other receptors are also responsible for virus entry into susceptible cells. In this study, we found that first-trimester trophoblast cells were resistant to RuV. In addition, we showed that $\mathrm{HaCaT}$ cells (an immortalized keratinocyte cell line) that did not express MOG on their surface were infected with RuV. This finding is one of the first demonstrations of MOG-independent RuV infection of susceptible host cells and suggests that it is important to continue searching for alternative RuV receptors. In addition, this study reports the resistance of first-trimester trophoblast cells to RuV and suggests that utilizing an epithelial-mesenchymal transition approach to study the mechanisms of transplacental vertical RuV infection.
\end{abstract}

Keywords: rubella; first trimester; trophoblasts; receptor; keratinocytes; infection

\section{Introduction}

Rubella virus (RuV) is an enveloped positive-strand RNA virus in the family Togaviridae and genus Rubivirus that causes mild symptoms such as rash and fever in children and young adults. However, infections in pregnant women, especially during the first trimester, can lead to dire consequences for the developing fetus, known as congenital rubella syndrome. The mechanisms by which RuV infects the fetus are not completely understood. In addition, many questions related to mechanisms of virus entry into susceptible host cells remain unanswered. Myelin oligodendrocyte glycoprotein (MOG) is reported to be a cellular receptor for RuV [1]; however, it is mainly expressed in the central nervous system and is barely detectable in other organs and tissues. Therefore, it is thought that other receptors are also responsible for virus entry into susceptible host cells [1,2].

While conducting our long-term research focused on the mechanisms of transplacental vertical $\mathrm{RuV}$ infection during the first trimester of pregnancy, we became interested in the role of the well-known protein MOG as a receptor for RuV and wondered if first-trimester trophoblast cells are susceptible to $\mathrm{RuV}$. We found that first-trimester trophoblast cells were resistant to RuV. In addition, we showed that HaCaT keratinocytes that did not express MOG on their surface were infected with 
$\mathrm{RuV}$. This finding is one of the first demonstrations of MOG-independent RuV infection of susceptible host cells and suggests that it is important to continue to search for alternative RuV receptors.

\section{Results and Discussion}

In this study, we found that the first-trimester trophoblast cell lines HTR-8/SVneo and Swan71 were resistant to both vaccine (Takahashi strain) and clinical (3-B1-RK13 strain) RuV strains, while keratinocytes (HaCaT) showed susceptibility to the virus (Figure 1). Nevertheless, MOG was not detected in any of the studied cells by indirect immunofluorescence assays and Western blotting, including human umbilical vein endothelial cells (HUVECs), which were previously reported to be permissive to $\mathrm{RuV}$ [3]. In addition, MOG mRNA was not detected by RT-PCR (Figure 2).

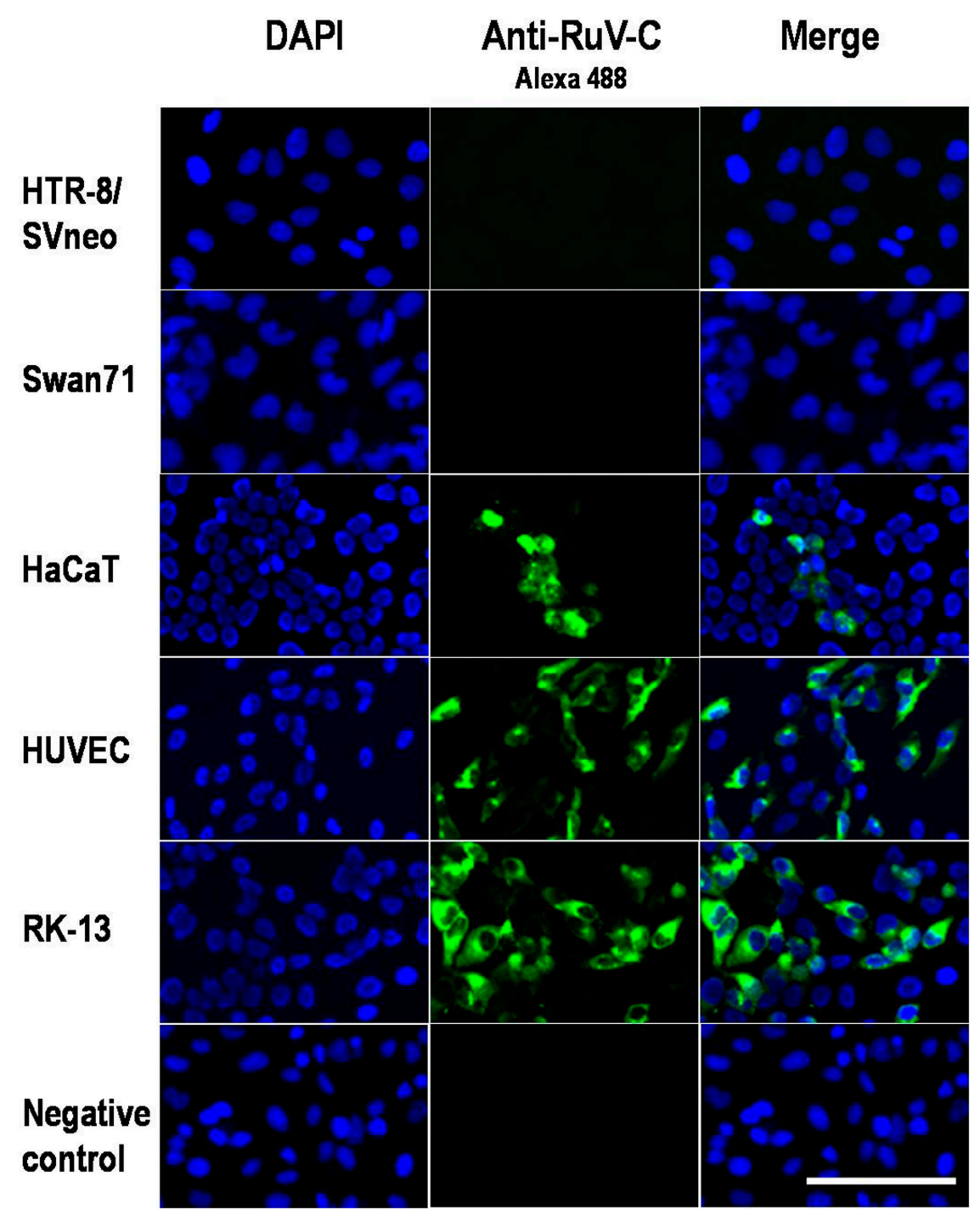

Figure 1. Microscopic images of cells infected or not infected with RuV. The cells were labeled with a mouse monoclonal anti-rubella virus capsid antibody (ab34749, Abcam, Tokyo, Japan) followed by a FITC-conjugated rabbit anti-mouse IgG $(\mathrm{H}+\mathrm{L}$ ) secondary antibody (green); nuclei were stained with DAPI (4',6-diamidino-2-phenylindole dihydrochloride) (blue). RuV-infected RK-13 cells and HUVECs were used as positive controls, and RuV-infected RK-13 cells stained with mouse serum were used as a negative control. No significant differences were noted on the infectivity of clinical and vaccine $\mathrm{RuV}$ strains to the studied cells by immunofluorescence assay. Images are representative of at least three independent experiments. RuV-C, rubella virus capsid. Scale bar: $100 \mu \mathrm{m}$. 


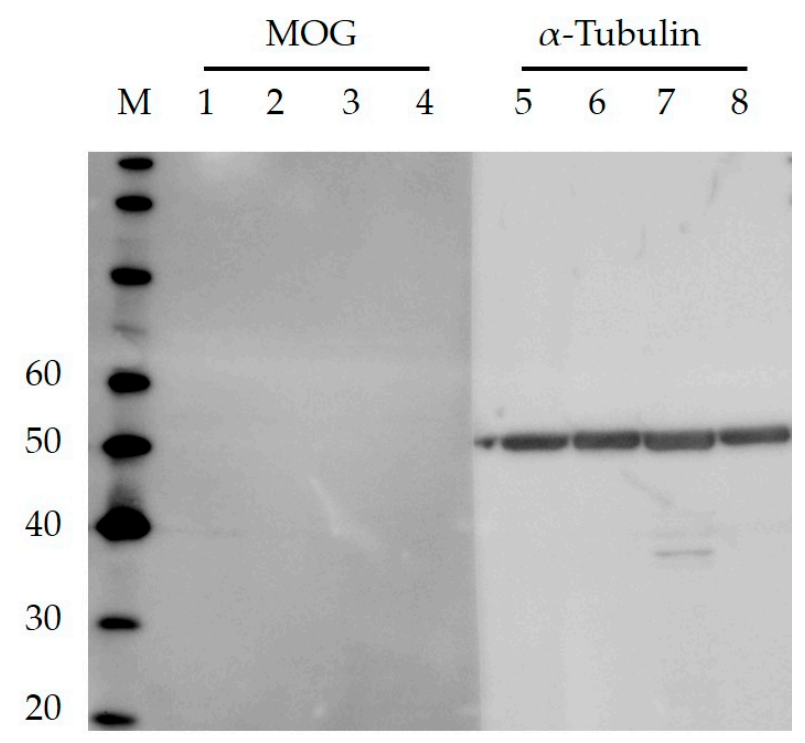

(a)

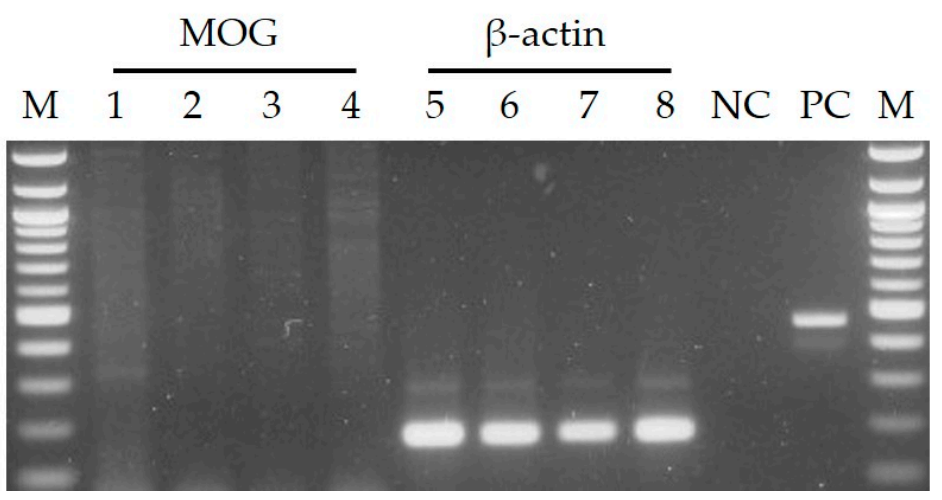

(b)

Figure 2. Investigation of MOG expression on first-trimester trophoblast cell lines, HaCaT and HUVECs. Images are representative of at least three independent experiments. (a) Western blot analysis. M, molecular weight marker (MagicMark XP Western Protein Standard, Invitrogen, Tokyo, Japan). Lanes 1-4 (for MOG, expected size: $28 \mathrm{kDa}$ ) and 5-8 (for $\alpha$-Tubulin), HTR8/SVneo, Swan71, HaCaT, HUVEC. (b) RT-PCR. M, 100 bp marker (TaKaRa, Shiga, Japan); lanes 1-4 (MOG, expected size: 218 bp) and 5-8 (for $\beta$-actin, 186 bp), HTR8/SVneo, Swan71, HaCaT, HUVEC; NC, negative control (RNAse-free water); PC, positive control (mRNA and corresponding primers provided in the RT-PCR Kit).

$\mathrm{RuV}$ was isolated from the skin of rubella patients [4,5]. It has been shown to cause persistent infections in keratinocytes, forming granuloma lesions in patients with primary immunodeficiency [6]. In this study, RuV could infect HaCaT cells, as well as HUVECs, that did not express MOG on their surface. Therefore, receptors other than MOG must be required for virus entry and replication in these cells. Otsuki and colleagues have recently published their findings showing that RuV has two distinct binding mechanisms: a $\mathrm{Ca}^{2+}$-dependent mechanism observed in lymphoid cells involving a direct interaction between $\mathrm{RuV}$ E1 protein and sphingomyelin/cholesterol-enriched membranes, and $\mathrm{a} \mathrm{Ca}^{2+}$-independent mechanism involving unidentified $\mathrm{RuV}$ receptor(s) [7]. Together with the above findings, this study suggests that it is necessary to continue searching for alternative $\mathrm{RuV}$ receptors.

It has been well established that transplacental transmission of rubella occurs in up to $90 \%$ of cases during the first eight weeks of gestation, falling to as low as $25 \%$ during the second trimester. Though most studies attribute fetal susceptibility to RuV-related teratogenesis in the first trimester of 
pregnancy to the critical periods of major organogenesis, structural changes in human placental tissues are another factor involved in utero transmission. This fluctuating incidence of fetal infection is likely related to changes in histological and molecular factors associated with placentogenesis. Therefore, considering the finding of this study, an epithelial-mesenchymal transition approach to studying the mechanisms of transplacental vertical RuV infection may be useful.

\section{Materials and Methods}

\subsection{Cells and Viruses}

\subsubsection{Cells and Cell Cultures}

The human first-trimester trophoblast cell lines Swan71 (derived from telomerase-mediated transformation of a seven-week cytotrophoblast isolate described by Straszewski-Chavez) [8] and HTR8/SVneo (originally obtained from human first-trimester placenta and immortalized by transfection with a cDNA construct that encodes the simian virus 40 large $\mathrm{T}$ antigen) [9] were cultured in Dulbecco's modified Eagle's medium (DMEM) (Swan71) or Roswell Park Memorial Institute 1640 normal growth medium (RPMI 1640) (HTR8/SVneo) (Gibco-Invitrogen, Tokyo, Japan) supplemented with $10 \%$ fetal bovine serum (FBS), $10 \mathrm{mM}$ HEPES, $0.1 \mathrm{mM}$ minimal essential medium non-essential amino acids, and $1 \mathrm{mM}$ sodium pyruvate (Gibco-Invitrogen). HaCaT cells were kindly provided by Dr. N.E. Fusenig (German Cancer Research Center, Heidelberg, Germany) and grown in DMEM containing 10\% FBS. Human umbilical vein endothelial cells (HUVECs; Lonza, Tokyo, Japan) were cultured in endothelial growth medium (2\% FBS) (EGM-2, Lonza). RK-13 cells were obtained from the Kitasato University School of Medicine (Tokyo, Japan) and were cultured in MEM supplemented with $10 \%$ FBS. All the above media were supplemented with $100 \mathrm{IU} / \mathrm{mL}$ penicillin and $100 \mu \mathrm{g} / \mathrm{mL}$ streptomycin. The cells were cultured in monolayers at $37{ }^{\circ} \mathrm{C}$ in a humidified $5 \% \mathrm{CO}_{2}$ incubator.

\subsubsection{Virus and Virus Infection}

$\mathrm{RuV}$ strains, both vaccine (Takahashi strain) and clinical (3-B1-RK13 strain), were obtained from Kitasato University School of Medicine (Tokyo, Japan). The viruses were propagated and titered in RK-13 cells.

Cells cultured on glass coverslips in six-well plates $\left(2 \times 10^{5}\right.$ cells/well $)$ were washed with phosphate buffered saline (PBS) and infected with RuV for $1 \mathrm{~h}$ at room temperature (RT). After a $1 \mathrm{~h}$ adsorption, appropriate medium supplemented with $\%$ FBS, either RPMI (for HTR8/SVneo cells) or DMEM (for Swan71 and HaCaT cells), was added, and the cells were incubated at $35^{\circ} \mathrm{C}$ in a humidified $5 \% \mathrm{CO}_{2}$ incubator. The next day, the supernatant was removed and replaced with fresh medium ( $2 \%$ FBS). Two days post-infection, the cells were subjected to immunofluorescence assays.

\subsection{Immunofluorescence Assay}

Cells grown on glass cover slips in six-well plates were incubated with $\mathrm{RuV}$ as described above. At $48 \mathrm{~h}$ post-infection, the supernatant was removed, and the cells were fixed with $4 \%$ paraformaldehyde (PFA) solution for $10 \mathrm{~min}$, washed with PBS, and incubated with a mouse monoclonal anti-RuV capsid antibody (ab34749, Abcam, Tokyo, Japan) for $1 \mathrm{~h}$ at RT. Negative controls were mock-treated with virus treatment and stained with normal mouse serum. After washing with PBS, the cells were incubated with a FITC-conjugated rabbit anti-mouse IgG $(\mathrm{H}+\mathrm{L})$ secondary antibody (Life Technologies, Tokyo, Japan) solution for $30 \mathrm{~min}$ at RT. The samples were counterstained with 4',6-diamidino-2-phenylindole dihydrochloride (DAPI) (Lonza) for nuclear staining. After washing, the cells were mounted with Fluoromount G (SouthernBiotech, Birmingham, AL, USA), and fluorescence images were collected using a fluorescence microscope (Floid Cell Imaging Station; Life Technologies, Tokyo, Japan). 
To examine MOG expression, cells grown on glass cover slips in six-well plates for $24 \mathrm{~h}$ were fixed with $4 \%$ PFA and then subjected to the procedures described above using a rabbit monoclonal anti-MOG antibody (ab109746, Abcam) followed by an Alexa Fluor 488-conjugated secondary antibody.

\subsection{Western Blotting}

Cells grown in six-well plates were incubated with $\mathrm{RuV}$ as described above. At $48 \mathrm{~h}$ post-infection, the supernatant was removed, and the cells were washed and then lysed in $70 \mu \mathrm{L}$ of cell lysis buffer (Cell Signaling Technology, Tokyo, Japan). The protein concentrations of the lysates were quantified using DC Protein Assay (Bio-Rad Laboratories, Inc., Hercules, CA, USA). The cell lysates were then loaded onto a NUPAGE 4-12\% Bis-Tris protein gel (Invitrogen) and separated by electrophoresis. Following electrophoresis, the proteins were transferred to polyvinylidene fluoride membranes (Invitrogen), and non-specific binding sites were blocked with 1\% bovine serum albumin in PBS with $0.1 \%$ Tween-20. The membranes were incubated with a mouse monoclonal anti-RuV capsid antibody at $4{ }^{\circ} \mathrm{C}$ overnight. The membrane was then incubated with horseradish peroxidase-conjugated secondary antibodies (Abcam) for $30 \mathrm{~min}$ at RT and visualized with a Luminescent Image Analyzer, Image Reader LAS-4000 mini (Fujifilm K. K, Tokyo, Japan). To examine MOG expression, cells grown in six-well plates for $24 \mathrm{~h}$ were subjected to the procedures described using a rabbit monoclonal anti-MOG antibody (ab109746, Abcam).

\subsection{Reverse Transcriptase PCR}

Total RNA was extracted from cells by using TRIzol (Invitrogen). The RNA concentration was quantified by using a nanodrop spectrophotometer at $260 \mathrm{~nm}$. RT-PCR was performed using PrimeScript One Step RT-PCR Kit Version 2 (Takara Bio Inc., Otsu, Japan) and the following primers: for MOG: sense, $5^{\prime}$-TCC TCC TCC TCC TCC AAG TGT CT-3'; antisense, $5^{\prime}$-AGT GGG GAT CAA AAG TCC GGT GG-3'; for $\beta$-actin: sense, 5'-TGG CAC CCA GCA CAA TGA A-3'; antisense, $5^{\prime}$-CTA AGT CAT AGT CCG CCT AGA AGC A-3' ${ }^{\prime}$. The PCR products were identified by electrophoresis on $1.5 \%$ agarose gels.

\section{Conclusions}

This study provides evidence of MOG-independent RuV infection and demonstrates that it is important to continue searching for alternative $\mathrm{RuV}$ receptors. As indicated by the susceptibility of $\mathrm{HaCaT}$ cells to $\mathrm{RuV}$, this study shows that an immortalized keratinocyte cell line can be used as an in vitro model for future studies of RuV. In addition, this study reports the resistance of first-trimester trophoblast cells to RuV and suggests utilizing an epithelial-mesenchymal transition approach to study the mechanisms of transplacental vertical RuV infection.

Acknowledgments: This study was supported by Grants-in-Aid for Scientific Research under the Japan Society for the Promotion of Science (JSPS KAKENHI grant numbers 17H04341 to Satoshi Hayakawa and 16K11160 to Quang Duy Trinh).

Author Contributions: Quang Duy Trinh, Shihoko Komine-Aizawa, and Satoshi Hayakawa conceived and designed the experiments; Quang Duy Trinh and Ngan Thi Kim Pham performed the experiments; Quang Duy Trinh and Kazuhide Takada analyzed the data; Quang Duy Trinh and Satoshi Hayakawa wrote the paper.

Conflicts of Interest: The authors declare no conflict of interest.

\section{References}

1. Cong, H.; Jiang, Y.; Tien, P. Identification of the myelin oligodendrocyte glycoprotein as a cellular receptor for rubella virus. J. Virol. 2011, 85, 11038-11047. [CrossRef] [PubMed]

2. Haralambieva, I.H.; Lambert, N.D.; Ovsyannikova, I.G.; Kennedy, R.B.; Larrabee, B.R.; Pankratz, V.S.; Poland, G.A. Associations between single nucleotide polymorphisms in cellular viral receptors and attachment factor-related genes and humoral immunity to rubella vaccination. PLoS ONE 2014, 9, e99997. [CrossRef] [PubMed] 
3. Perelygina, L.; Zheng, Q.; Metcalfe, M.; Icenogle, J. Persistent infection of human fetal endothelial cells with rubella virus. PLoS ONE 2013, 8, e73014. [CrossRef] [PubMed]

4. Heggie, A.D. Pathogenesis of the rubella exanthem. Isolation of rubella virus from the skin. N. Engl. J. Med. 1971, 285, 664-666. [CrossRef] [PubMed]

5. Marshall, W.C.; Trompeter, R.S.; Risdon, R.A. Letter: Chronic rashes in congenital rubella: Isolation of virus from skin. Lancet 1975, 1, 1349. [CrossRef]

6. Perelygina, L.; Plotkin, S.; Russo, P.; Hautala, T.; Bonilla, F.; Ochs, H.D.; Joshi, A.; Routes, J.; Patel, K.; Wehr, C.; et al. Rubella persistence in epidermal keratinocytes and granuloma M2 macrophages in patients with primary immunodeficiencies. J. Allergy Clin. Immunol. 2016, 138, 1436-1439. [CrossRef] [PubMed]

7. Otsuki, N.; Sakata, M.; Saito, K.; Okamoto, K.; Mori, Y.; Hanada, K.; Takeda, M. Both sphingomyelin and cholesterol in the host cell membrane are essential for Rubella virus entry. J. Virol. 2017, in press. [CrossRef] [PubMed]

8. Straszewski-Chavez, S.L.; Abrahams, V.M.; Alvero, A.B.; Aldo, P.B.; Ma, Y.; Guller, S.; Romero, R.; Mor, G. The isolation and characterization of a novel telomerase immortalized first trimester trophoblast cell line, Swan71. Placenta 2009, 30, 939-948. [CrossRef] [PubMed]

9. Graham, C.H.; Hawley, T.S.; Hawley, R.G.; MacDougall, J.R.; Kerbel, R.S.; Khoo, N.; Lala, P.K. Establishment and characterization of first trimester human trophoblast cells with extended lifespan. Exp. Cell Res. 1993, 206, 204-211. [CrossRef] [PubMed]

(C) 2018 by the authors. Licensee MDPI, Basel, Switzerland. This article is an open access article distributed under the terms and conditions of the Creative Commons Attribution (CC BY) license (http:/ / creativecommons.org/licenses/by/4.0/). 